\title{
The Luminosity Dependence of Quasar Clustering
}

\section{Citation}

Lidz, Adam, Philip F. Hopkins, Thomas J. Cox, Lars Hernquist, and Brant Robertson. 2006. "The Luminosity Dependence of Quasar Clustering." The Astrophysical Journal 641 (1): 41-49. https:// doi.org/10.1086/500444.

\section{Permanent link}

http://nrs.harvard.edu/urn-3:HUL.InstRepos:41381643

\section{Terms of Use}

This article was downloaded from Harvard University's DASH repository, and is made available under the terms and conditions applicable to Other Posted Material, as set forth at http:// nrs.harvard.edu/urn-3:HUL.InstRepos:dash.current.terms-of-use\#LAA

\section{Share Your Story}

The Harvard community has made this article openly available.

Please share how this access benefits you. Submit a story.

\section{Accessibility}


July 4, 2018. To BE SUbmitTed to ApJ.

Preprint typeset using $\mathrm{LAT}_{\mathrm{EX}}$ style emulateapj v. 04/03/99

\title{
THE LUMINOSITY DEPENDENCE OF QUASAR CLUSTERING
}

\author{
Adam Lidz ${ }^{1}$, Philip F. Hopkins ${ }^{1}$, Thomas J. Cox ${ }^{1}$, Lars Hernquist ${ }^{1}$, Brant Robertson ${ }^{1}$ \\ ${ }^{1}$ Harvard-Smithsonian Center for Astrophysics, 60 Garden Street, Cambridge, MA 02138, USA \\ July 4, 2018. To be submitted to ApJ.
}

\begin{abstract}
We investigate the luminosity dependence of quasar clustering, inspired by numerical simulations of galaxy mergers that incorporate black hole growth. These simulations have motivated a new interpretation of the quasar luminosity function. In this picture, the bright end of the quasar luminosity function consists of quasars radiating nearly at their peak luminosities, while the faint end consists mainly of very similar sources, but at dimmer phases in their evolution. We combine this model with the statistics of dark matter halos that host quasar activity. We find that, since bright and faint quasars are mostly similar sources seen in different evolutionary stages, a broad range in quasar luminosities corresponds to only a narrow range in the masses of quasar host halos. On average, bright and faint quasars reside in similar host halos. Consequently, we argue that quasar clustering should depend only weakly on luminosity. This prediction is in qualitative agreement with recent measurements of the luminosity dependence of the quasar correlation function (Croom et al. 2005) and the galaxy-quasar cross-correlation function (Adelberger \& Steidel 2005). Future precision clustering measurements from SDSS and 2dF, spanning a large range in luminosity, should provide a strong test of our model.
\end{abstract}

Subject headings: cosmology: theory - cosmology: observation - quasars: formation - large scale structure

\section{INTRODUCTION}

Recently, black hole growth and feedback have been incorporated into numerical simulations of galaxy mergers (Springel et al. 2005a,b). In these simulations, gravitational torques drive inflows of gas into the nuclei of merging galaxies (e.g. Barnes \& Hernquist 1991, 1996), triggering starbursts (e.g. Mihos \& Hernquist 1996) and feeding the growth of central supermassive black holes (Springel et al. 2005a; Di Matteo et al. 2005). As the black holes accrete, some of the radiated energy couples to the surrounding gas, and the growth eventually stalls when this feedback energy is sufficient to unbind the surrounding reservoir of gas. These simulations elucidate the connection between galaxy evolution, the formation of supermassive black holes, and the self-regulated nature of quasar activity, and provide quantitative predictions which agree well with observations of, e.g., the $M_{\mathrm{BH}}-\sigma$ relation (Di Matteo et al. 2005, Robertson et al. 2005), quasar lifetimes (Hopkins et al. 2005a,b), and the quasar luminosity function in various wavebands (Hopkins et al. 2005c,d,e). One important product of these numerical models is a quantitative prediction of the light curve of quasar activity and the resulting quasar "lifetime" - i.e., the amount of time that a quasar spends at a given luminosity - and its dependence on the properties of the merging galaxies.

The simulated quasar light curves imply a qualitatively different picture of the quasar luminosity function than previously considered. Specifically, in the model of Hopkins et al. (2005a-e), the bright end of the quasar luminosity function consists of systems radiating at close to their peak luminosities. The faint end of the luminosity function is dominated by similar quasars, observed, however, in a faint stage of their life cycle. This occurs because the quasar lifetime in this model is longer at lower luminosities; i.e. a given quasar spends more time (and is more likely to be observed) at a luminosity well below its peak luminosity. This differs from previous models which gen- erally assume that quasars radiate at a fixed luminosity for some characteristic lifetime, or adopt simplified exponential light curves (e.g., Kauffmann \& Haehnelt 2000, Wyithe \& Loeb 2003).

One way to distinguish our picture from previous models is through its predictions for the luminosity dependence of quasar clustering. In most semi-analytic models to date, there is assumed to be a tight relation between the instantaneous luminosity of a quasar and the mass of its host halo. In these analyses, faint quasars populate low mass halos, and are less clustered than bright quasars which populate high mass halos. In the model of Hopkins et al. (2005a-e) most bright and faint quasars are similar sources, seen at a different stage in their evolution. Therefore, one expects quasar clustering to depend less strongly on luminosity in this scenario than in most previous models. Although Hopkins et al. (2005e) demonstrate that this theory reproduces well a wide range of quasar observations not explained by previous models of the quasar light curve, the luminosity dependence of quasar clustering provides a direct probe of the most fundamental distinction between these models, and can be tested even at high redshift where observations of e.g. the Eddington ratio distribution of quasars are not currently practical.

In fact, Adelberger \& Steidel (2005) have recently measured the galaxy-quasar cross correlation function, finding no evidence for luminosity-dependent clustering. Their interpretation of this observation is that faint quasars are longer-lived than bright quasars. While this interpretation is qualitatively similar to the one we advocate, we will further demonstrate that it is, in fact, a natural consequence of our numerical models.

The aim of the present paper is to provide quantitative predictions for the luminosity dependence of quasar clustering, based on our numerical simulations. Our analysis proceeds in two steps. The first, described in $\S 2$, characterizes the relationship between quasar luminosity and the 
mass of quasar host dark matter halos. We use the numerical simulations of Springel et al. (2005a) to formulate our description of the connection between quasar luminosity and halo mass. The next step of our analysis is to connect quasar properties with the statistics of the dark matter halos that host quasars, as has been done previously (e.g, Efstathiou \& Rees 1998; Kauffmann \& Haehnelt 2000; Martini \& Weinberg 2001; Haiman \& Hui 2001; Porciani et al. 2004; Wyithe \& Loeb 2005). This part of our calculation is described in $\S 3$, where we determine which dark matter halos host active quasars, and provide predictions for the luminosity dependence of quasar bias. In $\S 4$ we explore the redshift evolution of quasar clustering. In $\S 5$ we conclude and summarize the present status of, and future prospects for, measurements of the luminosity dependence of quasar clustering.

\section{THE RELATION BETWEEN QUASAR LUMINOSITY AND HALO MASS}

We begin by connecting quasar luminosity with the mass of quasar host halos. The key point here is that the halo mass is correlated with the peak luminosity of quasar sources, and is connected only indirectly with the instantaneous luminosity through the quasar light curve. We therefore separate the connection between instantaneous quasar luminosity and halo mass into two distinct pieces. The first part is the correlation between peak luminosity and halo mass. The second part involves the quasar light curve which connects the instantaneous and peak luminosities of quasar activity. We illustrate this by evaluating the correlation between halo mass and peak luminosity, considering numerical simulations of merging galaxies at $z=2$, i.e., close to the epoch of peak quasar activity.

Specifically, we consider 24 simulations of merging galaxies at $z=2$. The simulations, described in more detail in Springel et al. (2005a) and Robertson et al. (2005), model merging galaxies of varying halo mass, initial disk gas fraction, and effective equation of state for the interstellar gas. In each simulation, we determine the amount of time the merger spends in each of several logarithmic bins in bolometric luminosity. We then determine the peak bolometric luminosity of a particular merger by identifying the highest luminosity bin reached. The peak bolometric luminosity is converted into a peak optical, B-band luminosity using the relation of Marconi et al. (2004), given by $\log _{10}\left(L_{B}\right)=0.80-0.067 \mathcal{L}+0.017 \mathcal{L}^{2}-0.0023 \mathcal{L}^{3}$, with $\mathcal{L}=\log _{10}\left(L_{\text {bol }} / L_{\odot}\right)-12$. Here, $L_{B}$ denotes the quasar B-band luminosity, while $L_{\text {bol }}$ indicates the quasar bolometric luminosity. ${ }^{1}$ The resulting peak B-band luminosity from our simulations, and its dependence on halo mass is shown in Fig. 1. The red open circles in the plot indicate the result of each merger simulation, while the cyan closed circles show the results (logarithmically) averaged over simulations with identical halo mass.

The plot indicates a clear correlation between the peak luminosities of quasars and the masses of their host dark matter halos. This correlation is a natural consequence of the self-regulated nature of quasar activity. Specifically, analytic models of self-regulated black hole growth predict that the peak luminosity scales with the halo circular

\footnotetext{
${ }^{1}$ In the remainder of the paper, we will use $L$ to denote the optical, B-band luminosity of quasar activity - i.e, we will generally suppress the subscript ' $\mathrm{B}$ '.
}

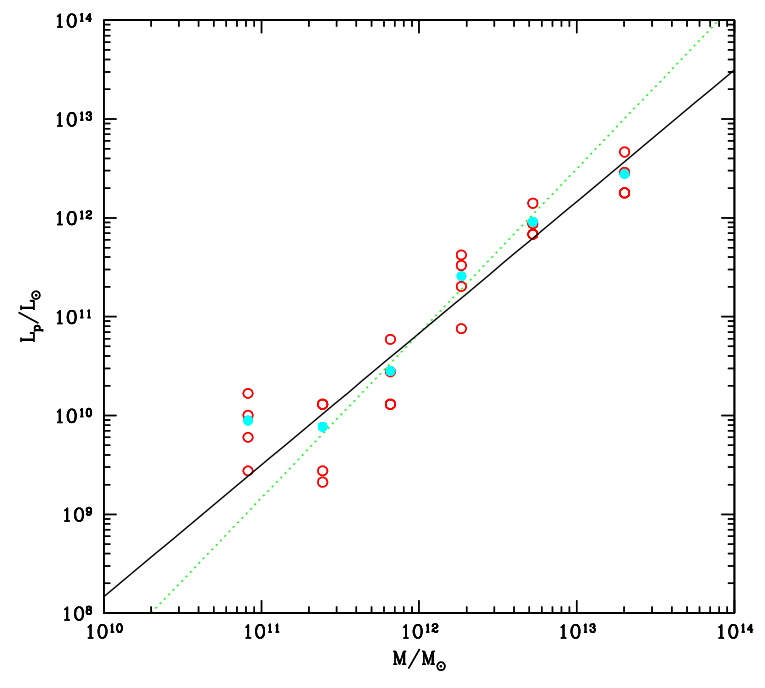

FIG. 1.- Correlation between peak B-band quasar luminosity and halo mass at $z=2$. The open red circles indicate the maximum, or peak B-band luminosity of quasar activity for each of 24 simulated halos at $z=2$ as a function of the mass of the quasar's host halo. The closed cyan circles indicate the mean B-band luminosity for quasars of a given halo mass. The green dotted line indicates the correlation expected from the model of Wyithe \& Loeb (2003). The black solid line indicates the correlation expected from analytic models of momentum-driven outflows (e.g., King 2003).

velocity, $v_{c}$, either as $L_{p} \propto v_{c}^{5}$, or as $L_{p} \propto v_{c}^{4}$. The first scaling is the result of assuming that the peak luminosity is set by equating the feedback energy from accretion coupled to the halo gas in a dynamical time with the binding energy of the gas (Silk \& Rees 1998; Ciotti \& Ostriker 2001; Wyithe \& Loeb 2002). The second scaling results from assuming that momentum, as opposed to energy, is conserved in the evolution of the quasar "outflow" that eventually unbinds the surrounding gas (e.g., King 2003, Di Matteo et al. 2005). This scaling is appropriate if the outflowing gas can cool efficiently.

Indeed, these authors suggest that this self-regulation likely explains the tight correlation observed between black hole mass and stellar velocity dispersion in local black hole populations (e.g. Gebhardt et al. 2000; Ferrarese \& Merritt 2000; Tremaine et al. 2002), with the peak luminosity corresponding to the Eddington luminosity of the final black hole mass. Ferrarese (2002) further argues that there is direct observational support for a correlation between black hole mass and halo circular velocity, as expected in the analytic models. Since the halo circular velocity is proportional to the one-third power of the halo mass, the first scaling implies that peak luminosity scales with halo mass as $L_{p} \propto M^{5 / 3}$, while the second scaling implies $L_{p} \propto M^{4 / 3}$.

A comparison between these scalings and the simulation results is shown in Fig. 1. The green dotted line indicates the first scaling, with the normalization adopted by Wyithe \& Loeb (2003) which, at $z=2$, is $L_{p}=6.78 \times 10^{10} L_{\odot}\left(M / 10^{12} M_{\odot}\right)^{5 / 3}$. The second scaling, $L_{p} \propto M^{4 / 3}$, with the same normalization, is indicated by 
the black solid line in the figure. Neither scaling is a perfect description of the mean trend seen in the simulations, although the somewhat shallower relation, $L_{p} \propto M^{4 / 3}$, is clearly a better overall match. This corresponds to a $M_{\mathrm{BH}}-\sigma$ relation of $M_{\mathrm{BH}} \propto \sigma^{4}$, (see Di Matteo et al. 2005, Robertson et al. 2005 for direct measurements of the $M_{\mathrm{BH}}-\sigma$ relation in our simulations), which agrees better with observations (e.g. Tremaine et al. 2002) than the alternate scaling. In practice, we find that halos with only a narrow range in mass host active quasars (see $\S 3$ ). Hence, we find that our results are quite similar if we use a direct spline fit to the mean simulated trend, (shown by the cyan circles in Fig. 1), or instead adopt the approximate $L_{p} \propto M^{4 / 3}$ scaling. Likewise, we have also analyzed in detail the difference between assuming an $L_{p} \propto M^{5 / 3}$ or $L_{p} \propto M^{4 / 3}$ scaling, and find that they give qualitatively identical results in our subsequent analysis. For simplicity, we therefore adopt the $L_{p} \propto M^{4 / 3}$ scaling in what follows.

In addition to the mean correlation between peak luminosity and halo mass, we would like to incorporate the amount of scatter in this relation into our modeling. It is clear from Fig. 1 that the level of scatter in our simulations is significant. Specifically, we find that the average dispersion in the peak luminosity at fixed halo mass is $\sigma_{\mathrm{L}_{\mathrm{p}}} / L_{p} \sim 0.8$. We account for this scatter, and the mean correlation between peak luminosity and halo mass, by adopting a lognormal form for the conditional probability distribution of peak luminosity given the halo mass,

$$
\frac{d P\left(L_{p} \mid M\right)}{d L_{p}}=\frac{1}{\sqrt{2 \pi \Delta^{2}} L_{p}} \operatorname{Exp}\left[-\frac{\left(\ln \left(\mathrm{L}_{\mathrm{p}} / \mathrm{L}_{\mathrm{m}}\right)\right)^{2}}{2 \Delta^{2}}\right],
$$

where $L_{m}$ denotes the mean peak luminosity for a halo of mass $M$. We adopt the scaling mentioned above, shown by the solid line in Fig. $1, L_{m}=6.78 \times 10^{10} L_{\odot}\left(M / 10^{12} M_{\odot}\right)^{4 / 3}$. The quantity $\Delta$ is the dispersion in the natural logarithm of the peak luminosity, $\Delta=\sigma_{\mathrm{L}_{\mathrm{p}}} / L_{p} \sim 0.8$ (0.35 dex, in good agreement with that observed by e.g. Marconi \& Hunt 2003).

\subsection{The Quasar Light Curve}

The next ingredients in our theoretical modeling are the quasar light curve and lifetime, which determine the amount of time that quasars with a given peak luminosity spend in different intervals in instantaneous luminosity. This represents the main difference between our modeling and previous work: in our picture, quasars spend an extended amount of time radiating at less than their peak luminosity, in contrast to models in which sources follow simplified 'on/off' ('light bulb') or pure exponential light curves. The observed quasar luminosity function should then be thought of as a convolution of the quasar light curve with an intrinsic distribution of sources of a given peak luminosity. We will follow Hopkins et al. (2005c,e) and extract the peak luminosity distribution from the observed luminosity function, using the quasar light curves obtained from our numerical simulations. Given the peak luminosity distribution, and the correlation between peak luminosity and halo mass described above, we can then predict the clustering properties of quasars.

We proceed by describing the relation between the quasar light curve, the peak luminosity distribution, and the quasar luminosity function. Specifically, the quasar luminosity function can be written as (Hopkins 2005e):

$$
L \frac{d \Phi}{d L}\left(t_{0}\right)=L \frac{d}{d L}\left[\int_{t_{0}-t_{q}\left(L \mid L_{p}\right)}^{t_{0}} d t^{\prime} \int \frac{d L_{p}}{L_{p}} L_{p} \frac{d \dot{n}_{p}\left(t^{\prime}\right)}{d L_{p}}\right],
$$

where $d \Phi\left(t_{0}\right) / d L$ denotes the luminosity function at (cosmic) time $t_{0}, t_{q}\left(L \mid L_{p}\right)$ represents the amount of time quasars with a given peak luminosity, $L_{p}$, spend at lower luminosities, $L$, and $d \dot{n}_{p} / d L_{p}$ is the rate at which quasars of a given $L_{p}$ are created or 'activated'. If we further assume that $d \dot{n}_{p} / d L_{p}$ is approximately constant over the lifetime of the quasar activity (as shown in Hopkins et al. 2005e), it follows that

$$
\begin{aligned}
L \frac{d \Phi}{d L}\left(t_{0}\right) & \sim \int \frac{d L_{p}}{L_{p}} L_{p} \frac{d \dot{n}_{p}\left(t_{0}\right)}{d L_{p}} L \frac{d t_{q}\left(L \mid L_{p}\right)}{d L} \\
& =\int \frac{d L_{p}}{L_{p}} L_{p} \frac{d n_{p}\left(t_{0}\right)}{d L_{p}} L \frac{d P\left(L \mid L_{p}\right)}{d L} .
\end{aligned}
$$

The second equality in the above equation further asserts that the product of the rate of producing quasars and the amount of time they spend at a given luminosity, is equal to the abundance of sources multiplied by the probability of finding an object at a given luminosity. This is justified by assuming that each quasar has a similar activation timescale, $t_{\mathrm{ac}}$, so that $\dot{n}_{p} \sim n_{p} / t_{\mathrm{ac}}$. The product of the lifetime, and the rate of producing quasars, $d t_{q}\left(L \mid L_{p}\right) / d L \times d \dot{n}_{p} / d L$, is then equal to $d P\left(L \mid L_{p}\right) / d L \times$ $d n_{p} / d L$, where the probability, $d P\left(L \mid L_{p}\right) / d L$, is the ratio of the lifetime to the activation timescale. Essentially, this is just a refinement of the commonly adopted proportionality between the probability of observing an object and its lifetime. We measure the quasar lifetime, $d t_{q}\left(L \mid L_{p}\right) / d L$, directly in our simulations, and so we know the above probability distribution up to a proportionality constant set by the activation timescale. In this paper we will not try to predict this proportionality constant theoretically, and hence our constraints come solely from the shape of the luminosity function, and not its absolute normalization. We then adopt the power law fitting formula for the quasar lifetime from Hopkins et al. (2005b):

$$
L_{\mathrm{bol}} \frac{d P\left(L_{\mathrm{bol}} \mid L_{\mathrm{p}, \mathrm{bol}}\right)}{d L_{\mathrm{bol}}} \propto|\alpha|\left(\frac{L_{\mathrm{bol}}}{10^{9} L_{\odot}}\right)^{\alpha}
$$

This applies for luminosities less than the peak luminosity, $L_{\mathrm{bol}}<L_{\mathrm{p}, \mathrm{bol}}$; it is zero otherwise. In this equation, $\alpha$ is a function of the peak bolometric luminosity, $L_{\mathrm{p}, \mathrm{bol}}$. Specifically, Hopkins et al. (2005b) give $\alpha=$ $\min \left[-0.2,-0.95+0.32 \log _{10}\left(\mathrm{~L}_{\mathrm{p}, \text { bol }} / 10^{12} \mathrm{~L}_{\odot}\right)\right]$. The above expressions are then converted from bolometric luminosity to optical B-band luminosity using the Marconi et al. (2004) formula, including a Jacobian factor to convert between the two differential probability distributions. ${ }^{2}$

We then invert Eq. 3 to find the distribution of quasar peak luminosities, $L_{p} d n_{\mathrm{p}} / d L_{p}$ (Hopkins et al. 2005c,e).

${ }^{2}$ We neglect here the obscuration effects modeled by Hopkins et al. (2005b,d,e) which relate the quasar light curves at different frequencies. We estimate that these effects are less important than uncertainties in the distribution of peak luminosities resulting from the poorly constrained faint end of the quasar luminosity function. 
In performing this inversion, we use the double power law fit from Boyle et al. (2000), to represent the observed luminosity function. ${ }^{3}$ We refer the reader to Hopkins et al. (2005c) for a plot of the resulting distribution, and present here only a qualitative description as follows. At high peak luminosity, the shape of the peak luminosity distribution tracks the shape of the observed luminosity function, it then reaches a peak near the break in the observed luminosity function, and turns over at low peak luminosity. The behavior of the distribution of peak luminosities simply reflects the fact that quasars with large peak luminosity spend long periods of time at low luminosity, and account, mostly by themselves, for the faint end of the quasar luminosity function: there is no need for quasars with small peak luminosity. The sharpness of the turnover in the peak luminosity distribution depends, however, on the poorly measured faint end of the quasar luminosity function. This behavior is insensitive to which of the many measured quasar luminosity functions we adopt, as demonstrated in Hopkins et al. (2005e).

\section{WHICH DARK MATTER HALOS HOST ACTIVE QUASARS?}

We now proceed to connect quasar properties with the properties of their host dark matter halos. Our motivation here is that the abundance and clustering of dark matter halos is well understood, and these quantities are easily extracted from numerical simulations of structure formation. Moreover, the results of detailed simulations (e.g. Springel et al. 2005c) agree well with analytic models based on an excursion set formalism in the context of an ellipsoidal collapse model (Sheth \& Tormen 2002). We can use these analytic models to specify the abundance and clustering of dark matter halos, and relate these to quasar properties. In this section we perform calculations at $z=2$ as an illustrative example; we generalize to other redshifts in $\S 4$.

In order to carry out this procedure, we adopt a simple phenomenological model: we assume that a fraction, $f_{\text {on }}(M)$, of halos of mass $M$ host active quasars. In the future, we will attempt to predict this quantity theoretically, but for now we will determine $f_{\text {on }}(M)$ from the distribution of peak luminosities above, the correlation between peak luminosity and halo mass from our simulations, and theoretical models for the abundance of dark matter halos. In what follows, we will use the mass function - i.e., the abundance of dark matter halos with mass between $M$ and $M+d M-$ derived by Sheth \& Tormen (2002), $d N_{\text {st }} / d M{ }^{4}$ The peak luminosity distribution is related to the halo mass function in our simple model by the relation

$$
L_{p} \frac{d n_{\mathrm{p}}}{d L_{p}}=\int d M \frac{d N_{\mathrm{st}}}{d M} f_{\text {on }}(M) L_{p} \frac{d P\left(L_{p} \mid M\right)}{d L_{p}} .
$$

In principle, this equation can be inverted to find $f_{\text {on }}(M)$. In practice, we instead adopt a lognormal form for $f_{\text {on }}(M)$, $f_{\text {on }}(M)=\left(\sqrt{2 \pi} \Delta_{m}\right)^{-1} \operatorname{Exp}\left[-\ln \left(\mathrm{M} / \mathrm{M}_{\mathrm{m}}\right)^{2} /\left(2 \Delta_{\mathrm{m}}^{2}\right)\right]$, and

${ }^{3}$ Specifically, we use the pure luminosity evolution fit in which the break magnitude is a quadratic function of redshift (Boyle et al. 2000).

${ }^{4}$ We perform all calculations with the Eisenstein \& Hu (1999) fitting formula for the transfer function, and adopt a cosmological model with the parameters $\Omega_{m}=0.3, \Omega_{\Lambda}=0.7, \Omega_{b} h^{2}=0.02$, and $\sigma_{8}(z=0)=0.9$.
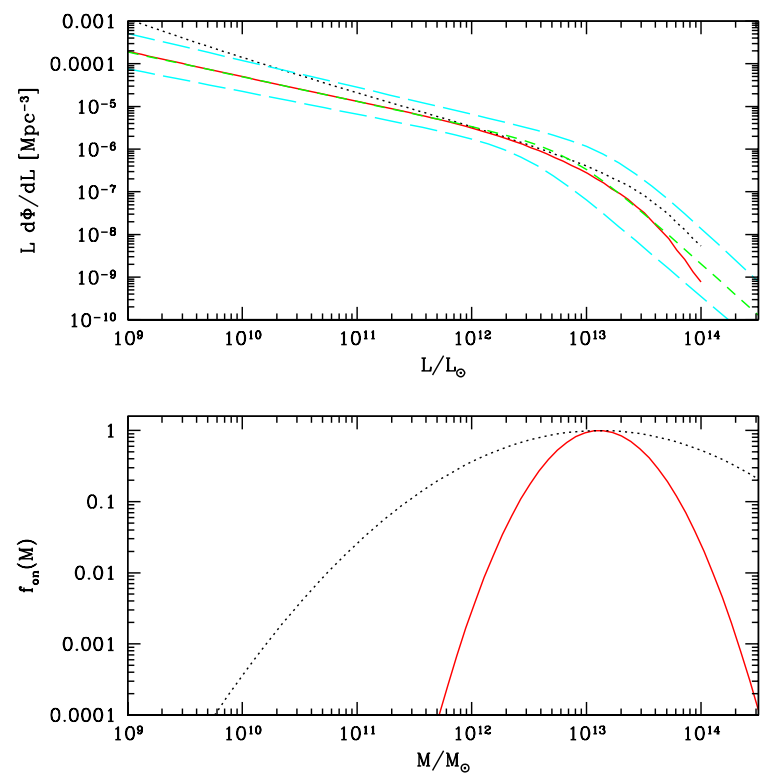

FIG. 2.- An indication of which dark matter halos house active quasars at $z=2$. The top panel shows the Boyle et al. (2000) fit to the $z=2$ quasar luminosity function, as well as two model predictions. The green dashed line represents the best fit double power law description of the data, and the cyan long-dashed lines indicate the allowed 1- $\sigma$ range. The solid red line and black dotted lines show model predictions. The bottom panel gives the corresponding fraction of halos of mass $M$ that host active quasars at $z=2$. The red solid line is favored by the data, and the quasar light curves from the simulations. The black dotted line shows a model that is a less good, but still gives an acceptable fit to the observations. The $\mathrm{y}$-axis in the bottom panel is not normalized.

determine the parameters of the lognormal, $\Delta_{m}, M_{m}$, that best match the distribution of peak luminosities, which is in turn constrained to match the observed luminosity function (see Eq. 3). The lognormal form is a convenient parameterization, since, as we will illustrate, our model predicts that quasar host halos have a well-defined characteristic mass. The peak of the lognormal distribution conveniently represents this characteristic mass, while the width of the distribution indicates how broad a range of halo masses host active quasars. As described in $\S 2$, we infer the peak luminosity distribution only up to an overall normalization constant, and so we are fitting only the shape of the $f_{\text {on }}(M)$ distribution, and not the absolute normalization.

The results of this exercise are shown in Fig. 2. The bottom panel of the figure shows two models for the fraction of halos of mass $M$ that host active quasars, while the top panel shows the corresponding predictions for the quasar luminosity function. The red solid line indicates the best fit, while the black dotted line describes a model that is an acceptable, but less good fit to the data. In our best fit model at $z=2$, active quasars are hosted by dark matter halos with a characteristic mass, $M \sim 1.3 \times 10^{13} M_{\odot}$. Moreover, only a narrow range of halo masses appear to house active quasars: the fractional width of the distribution $f_{\text {on }}(M)$ is only $\Delta_{m} \sim 0.75$. The narrowness of $f_{\text {on }}(M)$ is a reflection of the narrowness of the peak luminosity distribution seen in Hopkins et al. (2005c).

On the other hand, current measurements of the quasar 


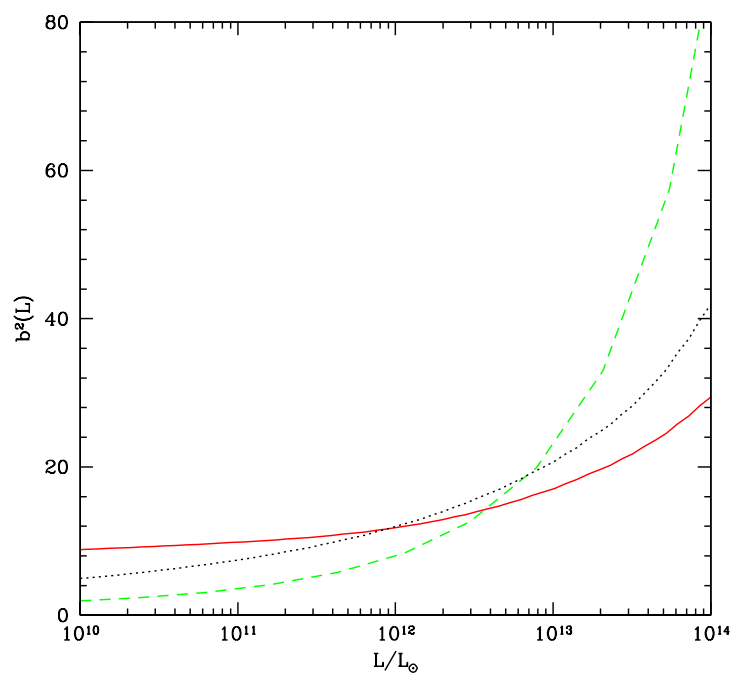

FIG. 3.- Bias-squared of quasars as a function of their luminosity at $z=2$. The red solid line shows the luminosity dependence of quasar clustering in our best-fit model (see the red solid line in Fig. 2 ). The black dotted line shows the same in our model which is a marginal fit to the quasar luminosity function (see the corresponding line in Fig. 2). Finally, the green dashed line shows the luminosity dependence of quasar clustering in a 'light bulb' model in which quasars radiate at their peak luminosity for their entire lifetime.

luminosity function do in principle accommodate a substantially broader $f_{\text {on }}(M)$ distribution. This is illustrated by the black dotted line in each panel of the figure. Here, the model luminosity function is normalized to match the observed luminosity function at a luminosity close to the break in the luminosity function. In this case, the characteristic mass of quasar host halos is the same as in our best fit model, while the fractional width of the distribution is $\Delta_{m} \sim 1.8$, nearly two and a half times as large as in our best fit model. This model is clearly a worse match to the Boyle et al. (2000) luminosity function, but it is still within the range allowed by the measurement errors. Finally, we note that if the mean peak luminosity, $L_{m}$, of Eq. 1 instead follows the Wyithe \& Loeb (2003) relation, $L_{m} \propto M^{5 / 3}$, the characteristic halo mass would be $\sim 7.5 \times 10^{12} M_{\odot}$, and the width of the distribution would be $\Delta_{m} \sim 0.5$.

To reiterate, quasars with large peak luminosity sit in massive halos, and spend a significant amount of time at lower luminosities. These sources already account for the faint end of the luminosity function, and hence one overproduces the abundance of faint quasars if low mass halos host active quasars. Moreover, if very massive halos house active quasars, one might over-produce the bright end of the quasar luminosity function. Consequently, a wide range of quasar luminosities corresponds to only a narrow range in host halo mass. The extent to which this is true depends on the details of the faint end of the quasar luminosity function, which is thus far poorly measured.

We can now turn our constraint on the fraction of halos of mass $M$ that host active quasars into a constraint on the luminosity dependence of quasar clustering. It will first be useful to write down an expression for the conditional probability that a halo of mass $M$ houses a quasar with instantaneous B-band luminosity $L$. This is analogous to the conditional luminosity function in the halo-occupation distribution formalism, considered in the context of the abundance and clustering of galaxies (e.g., Yang, Mo, \& van den Bosch 2003). This probability distribution can be written as

$$
L \frac{d P(L \mid M)}{d L}=f_{\text {on }}(M) \int \frac{d L_{p}}{L_{p}} L \frac{d P\left(L \mid L_{p}\right)}{d L} L_{p} \frac{d P\left(L_{p} \mid M\right)}{d L_{p}} .
$$

We can then express the luminosity function as an integral over halo mass. (This is in contrast to Eq. 3 where we expressed the quasar luminosity function as an integral over peak luminosity.) This relation is

$$
L \frac{d \Phi}{d L}=\int d M \frac{d N_{\mathrm{st}}}{d M} L \frac{d P(L \mid M)}{d L} .
$$

An expression for the bias of a quasar of instantaneous B-band luminosity, $L$, then follows in terms of the bias of a halo of mass $M,{ }^{5}$

$$
b(L)=\left[L \frac{d \Phi}{d L}\right]^{-1} \int d M \frac{d N_{\mathrm{st}}}{d M} b(M) L \frac{d P(L \mid M)}{d L} .
$$

To complete the calculation, we require an expression for the bias of a halo of mass $M, b(M)$. We use the formula from Sheth, Mo, \& Tormen (2001), which agrees well with measurements from N-body simulations.

We investigate the luminosity dependence of quasar clustering in three different models. The first two are each based on our simulated quasar light curves. These two correspond to the curves shown in Fig. 2 and are meant to bracket the possible range allowed by present luminosity function measurements. We contrast these models with one in which the instantaneous luminosity is related to the halo mass in the same way that the peak luminosity is related to the halo mass in our model, i.e., $L \propto M^{4 / 3}$. Furthermore, in this scenario we neglect any scatter in the relation between luminosity and halo mass. This is equivalent to the 'light bulb' model assumption in which quasars radiate at exactly their peak luminosity for their entire lifetime.

The results of this calculation are shown in Fig. 3, which illustrates the main point of this paper. The plot reveals significant differences between the three models. Our first model corresponds to the $f_{\text {on }}(M)$ distribution which best matches the observed luminosity function, as shown in Fig. 2. The bias in this model, denoted by the red solid line, is quite flat as a function of luminosity, before ramping up at very high luminosity. Our second model corresponds to the broader $f_{\text {on }}(M)$ distribution of Fig. 2, which provides a marginal match to the observed luminosity function. The

\footnotetext{
${ }^{5}$ The bias is defined so that, on large scales, the power spectrum of halos of mass $M$ is related to the dark matter power spectrum via $P_{M}(k)=b^{2} P_{\mathrm{dm}}(k)$. Here, $P_{M}(k)$ and $P_{\mathrm{dm}}(k)$ represent the halo and dark matter power spectra, respectively, at wavenumber $k$. We caution that this description applies only when the scale under consideration is much larger than the virial radius of the host halos (e.g., Scoccimarro et al. 2001.) Some care is therefore required when connecting observed correlation lengths with the bias of the source host halos.
} 
bias in this model, denoted by the black dotted line, is qualitatively similar to that in our best fit model, although it is a less flat function of luminosity. Finally, in the 'light bulb' model, the results are quite different: the quasar bias increases much more sharply with luminosity.

The difference between the bias in these models is easy to understand. In the case of the 'light bulb' scenario, there is a one-to-one relation between luminosity and halo mass. The range of luminosities shown in the plot thus corresponds to a wide range in halo mass. The quasar bias, which depends strongly on halo mass, increases sharply with luminosity. Considering a pure exponential light curve gives an essentially identical result, as the implied peak luminosity distribution has nearly the same shape (Hopkins et al. 2005c). In the case of our models based on realistic quasar light curves, however, the entire range in luminosity corresponds to only a relatively small range in halo mass. As a result, the variation of quasar bias with luminosity is rather weak. The red solid line and the black dotted line illustrate that exactly how weak the trend with luminosity is depends on how narrow the $f_{\text {on }}(M)$ distribution is, which is relatively poorly constrained by current luminosity function measurements.

One might wonder about the implications of these results for attempts to infer the lifetimes of quasars from their clustering properties (Martini \& Weinberg 2001; Haiman \& Hui 2001). The usual view is that quasar clustering makes it possible to distinguish whether quasars are numerous yet short-lived, or are rare but long-lived sources. Here we merely echo the sentiment of Adelberger \& Steidel (2005): quasar lifetimes depend strongly on the instantaneous luminosity of quasar activity and the 'duty cycle' is larger for faint objects than bright ones. By 'duty cycle', we mean the ratio of the abundance of quasars in a given luminosity range to the abundance of the dark matter halos that host them (Martini \& Weinberg 2001; Haiman \& Hui 2001; Adelberger \& Steidel 2005). Indeed, let us consider our model in which the characteristic halo mass at $z=2$ is $\sim 1.3 \times 10^{13} M_{\odot}$, and the dispersion in halo mass is $\Delta_{m} \sim 0.75$. In this case, the duty cycle for quasars with instantaneous B-band luminosity in the range $10^{10}-10^{11} L_{\odot}$ is quite large, $\sim 0.3$. On the other hand, quasars with luminosity in the range $10^{13}-10^{14} L_{\odot}$ have a duty cycle of only $\sim 8 \times 10^{-4}$. In other words, the quasar lifetime derived from quasar clustering should depend strongly on the luminosity of the sources considered, and should not be interpreted as an intrinsic lifetime (Hopkins et al. 2005a,e).

Finally, we note that our model predicts only the relative duty cycles of faint and bright quasars, since we do not attempt to predict the absolute normalization of the peak luminosity distribution. Although the bias as a function of luminosity is independent of this normalization, future work, incorporating theoretical estimates of the merger rates of gas rich galaxies, will be necessary to test whether these models can produce the large faint-quasar duty cycle implied by our best fit case. Alternatively, the $f_{\text {on }}(M)$ distribution may be less narrow than assumed above.

\section{REDSHIFT EVOLUTION}

It is also interesting to consider the redshift evolution of quasar clustering. The luminosity dependence of quasar clustering is poorly determined at each redshift we con- sider (although see Croom et al. 2005; Adelberger \& Steidel 2005), and hence measurements (integrated over all luminosities) at different redshifts do not currently provide a strong test of our contention that bright and faint quasars reside in similar mass host halos. However, it does provide a consistency test regarding our assertion that the observed break in the quasar luminosity function corresponds to a turnover in the peak luminosity distribution, and of our assumed correlation between peak luminosity and halo mass. Moreover, from Fig. 2, we expect that quasar host halos may have a well defined characteristic mass. It is natural then to ask how this characteristic mass evolves with redshift (e.g., Porciani et al. 2004; Croom et al. 2005, Wyithe \& Loeb 2005).

There are several factors that might drive redshift evolution in the characteristic mass of quasar host halos. In scenarios in which black hole growth is self-regulated by feedback, the final black hole mass is partly set by the depth of the gravitational potential well of the host halo. In this case, one expects the normalization of the relation between black hole mass and circular velocity to remain constant with redshift. The same is not true, however, for the relation between halo mass and black hole mass: high redshift halos of a given mass have deeper gravitational potential wells and can grow larger black holes than halos of the same mass at lower redshift (e.g. Wyithe \& Loeb 2003). More specifically, from the scaling $M_{\mathrm{bh}} \propto v_{c}^{4}$, and connecting circular velocity to halo mass, we have $M_{\mathrm{bh}} \propto[\zeta(z)]^{2 / 3}(1+z)^{2} M^{4 / 3}$ (Wyithe \& Loeb 2003), where $\zeta(z)=\left[\Omega_{m} / \Omega_{m}(z)\right]\left[\Delta_{c} / 18 \pi^{2}\right]$, and $\Delta_{c}=18 \pi^{2}+82\left(\Omega_{m}(z)-1\right)-39\left(\Omega_{m}(z)-1\right)^{2}$. Here, $\Omega_{m}(z)$ denotes the matter density at redshift $z$ in units of the critical density, and $\Delta_{c}$ is the collapse overdensity according to the fitting formula of Bryan \& Norman (1998). We further assume that the relation between peak luminosity and final black hole mass does not evolve with redshift, in which case the redshift evolution of the peak luminosity-halo mass relation is the same as that of the black hole mass-halo mass relation. These assumptions are further confirmed in numerical simulations at different redshifts, or more accurately, simulations in which we vary the properties of the merging galaxies to mimic redshift evolution (Robertson et al. 2005). Moreover, the break quasar luminosity, $L_{\star}$, evolves with redshift (e.g. Boyle et al. 2000), as does the shape of the halo mass function.

We aim, then, to piece together each of these evolving ingredients, and determine the evolution of quasar clustering with redshift, employing the same methodology as in $\S 2$ and $\S 3$. To this end, we adopt the pure luminosity evolution (PLE) double power law model of Boyle et al. (2000) in which the break magnitude varies quadratically with redshift, $M_{\star}(z)=-22.65+1.35 z-0.27 z^{2}$. For simplicity, we will further assume that the scatter in the relation between peak quasar luminosity and halo mass is independent of redshift. We can then infer the distribution of quasar peak luminosities (Eq. 3) and determine which dark matter halos host active quasars $\left(f_{\text {on }}(M)\right.$ in Eq. 5) at each of several redshifts. At each redshift, we try to determine the $f_{\text {on }}(M)$ that provides the best fit to the quasar luminosity function, noting that current luminosity function measurements tolerate a wide range of values for $f_{\text {on }}(M)$, as illustrated in Fig. 2. We expect 


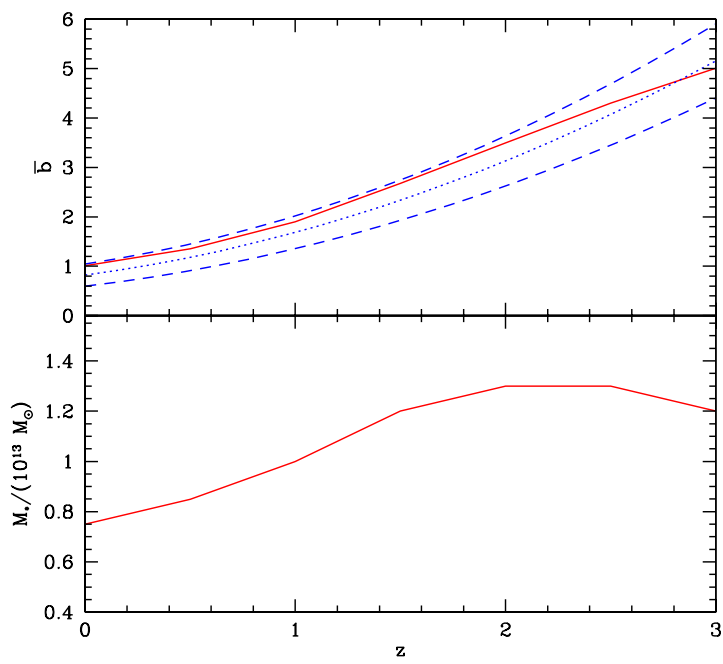

FIG. 4.- Quasar bias as a function of redshift. The top panel shows, as a red solid line, our theoretical expectation for the redshift evolution of quasar bias. The kink in the model prediction is likely an artifact of extending the PLE model of Boyle et al. (2000) beyond the redshift extent of their measurement. The blue dotted line shows the best fit measurement from Croom et al. (2005), while the blue dashed-lines indicate the allowed 1- $\sigma$ range implied by their measurement. The bottom panel shows the corresponding characteristic mass of dark matter halos that host active quasars as a function of redshift.

the qualitative feature illustrated in the previous section, that quasar bias depends weakly on luminosity, to hold at all redshifts. In order to illustrate the redshift evolution of the typical quasar bias, we then consider a luminosityaveraged quasar bias defined by

$$
\bar{b}=\left[\int_{L_{\min }}^{L_{\max }} d L \frac{d \Phi}{d L}\right]^{-1} \int_{L_{\min }}^{L_{\max }} d L b(L) \frac{d \Phi}{d L} .
$$

In what follows, we choose $L_{\min }=0.1 L_{\star}(z)$, and $L_{\max }=$ $10 L_{\star}(z)$. Although this does not correspond precisely to the quasar bias that is measured observationally (e.g., Croom et al. 2005), our results are not very sensitive to our choices for $L_{\min }$ and $L_{\max }$. After all, the bias in our model depends only weakly on luminosity.

The results of this calculation are shown in Fig. 4. The bottom panel of the figure shows the characteristic mass of quasar host halos - specifically, the center of the lognormal distribution $f_{\text {on }}(M)$ (Eq. 5) - for several redshifts. The figure clearly illustrates that the characteristic mass of quasar host halos evolves relatively little with redshift in our model. The reason for this is as follows. The characteristic mass of quasar host halos is primarily determined, within the context of our model, by the turnover in the distribution of quasar peak luminosities and the correlation between peak luminosity and halo mass. The turnover in the distribution of quasar peak luminosities is in turn set by the position of the break in the quasar luminosity function. The redshift evolution of the position of the break in the quasar luminosity function is, however, compensated by evolution in the peak luminosity-halo mass relation, and somewhat by changes in the shape of the halo mass function. Consequently, we find that our model produces the observed evolution in the break luminosity at close to fixed host halo mass: the characteristic host halo mass appears to vary by less than a factor of $\sim 2$ between $z=0$ and $z=3$. We note that the figure shows a turnover in the host halo mass near $z \sim 2.5$, which corresponds to a similar turnover in $L_{\star}(z)$, but this is likely an artifact of extending the PLE model of Boyle et al. (2000) beyond the redshift extent of their measurement $(z \sim 2.3)$. Finally, we remark that if the peak luminosity scales with halo mass as $L_{p} \propto M^{5 / 3}$ then the results are qualitatively similar, but the characteristic halo masses are a factor of $\sim 1.5-2$ times smaller.

The redshift evolution of the break luminosity is then a reflection of the self-regulated nature of quasar activity: halos that host quasars have deeper potential wells at high redshift and can thereby grow larger, more luminous black holes at high redshift than at low redshift. In this sense, black hole growth is anti-hierarchical: massive black holes form at higher redshifts than low-mass black holes (Cowie et al. 2003). In order to understand the physics that drives this anti-hierarchical growth in more detail, we need to understand what sets the characteristic mass of quasar host halos, a topic we briefly speculate on in the concluding section.

We show the resulting prediction for quasar bias as a function of redshift in the top panel of Fig. 4. We compare our theoretical predictions with the quasar bias measured by Croom et al. (2005). ${ }^{6}$ The figure illustrates that our theoretical predictions agree with the measured bias as a function of redshift, although our results are a bit higher the best fit measurements. This slight overprediction is somewhat sensitive to the assumed peak luminosityhalo mass relation, however, in the sense that assuming $L_{p} \propto M^{5 / 3}$ produces better agreement with the best fit measurements. The key qualitative feature of the figure is that, even though quasars at $z \sim 0$ and $z \sim 3$ reside in similar host halos, their clustering properties differ significantly. Specifically, quasars at $z \sim 0$ should be close to un-biased $(\bar{b} \sim 1)$, while quasars at $z \sim 3$ are highly biased, with $\bar{b} \sim 5$. The reason for this is simply that halos of mass $\sim 7.5 \times 10^{12} M_{\odot}-1.5 \times 10^{13} M_{\odot}$ correspond to rare, high- $\sigma$ peaks at $z \sim 3$, and are thus highly-clustered. On the other hand, the variance of the density field smoothed on the same mass scale is close to the collapse threshold at $z \sim 0, \sigma(M) \sim \delta_{c}$, and hence these halos faithfully trace the matter distribution (see also Croom et al. 2005, Wyithe \& Loeb 2005) near $z \sim 0$. This trend is to be expected in the context of our model if mergers involving gas-rich galaxies occur mainly in dense environments at high redshifts, but in more isolated regions at $z \sim 0$.

\section{CONCLUSION}

\footnotetext{
${ }^{6}$ Croom et al. (2005) derive the quasar bias assuming a slightly different cosmological model than we adopt here. The difference between the bias in our two models should, however, be small compared to the statistical errors in the measurement. In addition, the quantity Croom et al. (2005) measure is a little different from the luminosity-averaged bias we predict in Eq. 9. Again, the difference between our definitions of quasar bias should be small compared to statistical measurement errors.
} 
In this paper, we have connected the properties of quasars, as determined from numerical simulations of galaxy mergers (Springel et al. 2005a, Di Matteo et al. 2005, Robertson et al. 2005), to the properties of the dark matter halos that host them. We find that bright and faint quasars reside in similar mass halos with characteristic masses close to $\sim 1 \times 10^{13} M_{\odot}$. As a result, we predict that quasar clustering should depend only weakly on quasar luminosity. Furthermore, we predict that the characteristic mass of quasar host halos should evolve only weakly with redshift. We note that Di Matteo et al. (2003) also found, using cosmological simulations with a simple model for black hole growth, that quasars at low and high redshift reside in similar mass host halos, although they associate quasars with less massive halos than we find presently. Our conclusions invite two obvious questions. The first question is of a theoretical nature: what physics sets the characteristic mass scale of quasar host halos? The second question is an observational one: what do $o b$ servational measurements of the luminosity dependence of quasar clustering find?

We will largely defer answering the first question to future work, but a plausible explanation is that the most luminous quasars at a given redshift are triggered by the most massive gas rich galaxies merging at that time. The halos that host these objects will be determined by the evolution of merger rates, depending on environment, and the gas content of the galaxies they contain. We note that more precise luminosity function measurements will be helpful in obtaining tighter constraints on the width of the $L_{p}$ distribution, and on the mass range of halos that host active quasars. Observations of e.g. the Eddington ratio distribution and active black hole mass function can further constrain this distribution at faint luminosities where the observed luminosity function provides only weak limits (Hopkins et al. 2005e). Likewise, the distribution of host masses can be constrained by observations of the quasar host galaxy luminosity function; these find an approximately lognormal distribution with narrow width $\Delta_{L} \sim \Delta_{m}=0.5(\sim 0.6-0.7$ magnitudes $)$ and a peak corresponding to the stellar mass of quasar hosts with $L_{p} \sim L_{\star}$ (Bahcall et al. 1997; McLure et al. 1999; Hamilton et al. 2002), close to that predicted by our best-fit model. Further progress can be made theoretically with more detailed semi-analytic calculations incorporating galaxy merger rates (Kauffman \& Haehnelt 2002), or with cosmological simulations incorporating black hole growth and feedback.

We now address the second question. There have been two recent observational attempts to measure the luminosity dependence of quasar clustering. First, Croom et al. (2005) examine the luminosity dependence of quasar clustering from $\sim 20,0002 \mathrm{dF}$ quasars, finding no evidence for any luminosity dependence. Their measurement, however, spans only a factor of $\lesssim 20$ in luminosity. Second, Adelberger \& Steidel (2005) examine the luminosity dependence of quasar clustering using the galaxyAGN cross-correlation function, rather than the quasar auto-correlation function. This approach, initially advocated by Kauffmann \& Haehnelt (2002), takes advantage of the fact that galaxies are much more abundant than quasars: therefore, statistical measurements of the galaxy-
AGN cross correlation function are correspondingly tighter than measurements of the quasar auto-correlation function. Furthermore, Adelberger \& Steidel (2005) probe the luminosity dependence of quasar clustering with a much larger dynamic range, roughly a factor of 10 in magnitude, or a factor of $10^{4}$ in luminosity. Their result is, again, that quasar clustering is independent of luminosity. Specifically, they find that the correlation length for quasar sources with $-30<M_{1350}<-25$ is $r_{0}=4.7 \pm 2.3$, and $r_{0}=5.4 \pm 1.2$ for sources with $-25<M_{1350}<-19$, where $M_{1350}$ denotes an $\mathrm{AB}$ magnitude at a rest-frame wavelength of $1350 \AA$. The statistical precision of these results is not high, but again, they are qualitatively consistent with our picture. We eagerly anticipate more precise measurements from SDSS and $2 \mathrm{dF}$ in the near future, which should provide a more definitive test of this picture for quasar formation and evolution.

Finally, we remark that in this paper we confined our theoretical calculations to large scales where linear biasing is an accurate description of clustering, but it would be interesting to extend calculations to smaller scales. Indeed, Hennawi et al. (2005) find that the quasar correlation function is an order of magnitude larger, at proper separations of $r \lesssim 40 \mathrm{kpc} / \mathrm{h}$, than expected based on extrapolating clustering measurements from large scales. This is likely evidence that quasar clustering is associated with galaxy mergers in dense environments, but this should be quantified.

We are extremely grateful to Tiziana Di Matteo and Volker Springel for their role in the simulations which inspired this analysis, and for helpful comments on a draft. This work was supported in part by NSF grants ACI 96-19019, AST 00-71019, AST 02-06299, and AST 0307690, and NASA ATP grants NAG5-12140, NAG5-13292, and NAG5-13381. The simulations used in the analysis were performed at the Center for Parallel Astrophysical Computing at the Harvard-Smithsonian Center for Astrophysics.

\section{REFERENCES}

Adelberger, K., \& Steidel, C., 2005, astro-ph/0505210

Bahcall, J. N., Kirhakos, S., Saxe, D. H., \& Schneider, D. P. 1997, ApJ, 479, 642

Barnes, J.E. \& Hernquist, L. 1991, ApJ, 370, L65

Barnes, J.E. \& Hernquist, L. 1996, ApJ, 471, 115

Boyle, B. J., Shanks, T., Croom, S. M., Smith, R. J., Miller, L., Loaring, N., \& Heymans, C. 2000, MNRAS, 317, 1014

Bryan, G. L., \& Norman, M. 1998, ApJ, 495, 80

Ciotti, L., \& Ostriker, J. P., 2001, ApJ, 551, 131

Cowie, L. L., Barger, A. J., Bautz, M. W., Brandt, W. N., \& Garmire, G. P. 2003, ApJ, 584, L57

Croom, S. M. et al., 2005, MNRAS, 356, 415

Di Matteo, T., Croft, R. A. C., Springel, V., \& Hernquist, L. 2003, ApJ, 593, 56

Di Matteo, T., Springel, V., \& Hernquist, L., 2005, Nature, 433, 604

Efstathiou, G., Rees, M. J., 1988, MNRAS, 230, 5

Eisenstein, D. J., \& Hu, W., 1999, ApJ, 511, 5

Ferrarese, L., \& Merritt, D., 2000, ApJL, 539, 1

Ferrarese, L., 2002, ApJ, 578, 90

Gebhardt, K. et al., 2000, ApJ, 539, L13

Haiman, Z., \& Hui, L., 2001, ApJ, 547, 27

Hamilton, T. S., Casertano, S., \& Turnshek, D. A. 2002, ApJ, 576, 61

Hennawi, J. F. et al., 2005, AJ submitted, astro-ph/0504535

Hopkins, P. F., Hernquist, L., Martini, P., Cox, T. J., Robertson, B., Di Matteo, T., \& Springel, V., 2005a, ApJL in press, astro$\mathrm{ph} / 0502241$ 
Hopkins, P. F., Hernquist, L., Cox, T. J., Di Matteo, T., Martini, P., Robertson, B., \& Springel, V., 2005b, ApJ in press, astro$\mathrm{ph} / 0504190$

Hopkins, P. F., Hernquist, L., Cox, T. J., Di Matteo, T., Robertson, B., \& Springel, V., 2005c, ApJ in press, astro-ph/0504252

Hopkins, P. F., Hernquist, L., Cox, T. J., Di Matteo, T., Robertson, B., \& Springel, V., 2005d, ApJ in press, astro-ph/0504253

Hopkins, P. F., Hernquist, L., Cox, T. J., Di Matteo, T., Robertson, B., \& Springel, V., 2005e, ApJ submitted, astro-ph/0506398

Kauffmann, G., \& Haehnelt, M., 2000, MNRAS, 311, 576

Kauffmann, G., \& Haehnelt, M., 2002, MNRAS, 332, 529

King, A., 2003, ApJL, 596, 27

Marconi, A. et al., 2004, MNRAS, 351, 169

Marconi, A., \& Hunt, L. K. 2003, ApJ, 589, L21

Martini, P. \& Weinberg, D. H., 2001, ApJ, 547, 12

McLure, R. J., Kukula, M. J., Dunlop, J. S., Baum, S. A., O'Dea, C. P., \& Hughes, D. H. 1999, MNRAS, 308, 377

Mihos, J.C. \& Hernquist, L. 1996, ApJ, 464, 641

Porciani, C., Magliocchetti, M., \& Norberg, P., 2004, MNRAS, 355, 1010

Robertson, B., Hernquist, L., Cox, T. J., Di Matteo, T., Hopkins, P. F., Martini, P., \& Springel, V., 2005, ApJ, submitted, astro$\mathrm{ph} / 0506038$

Scoccimarro, R., Sheth, R. K., Hui, L., \& Jain, B., 2001, ApJ, 546, 20

Sheth, R. K., Mo, H. J., \& Tormen, G., 2001, MNRAS, 323, 1

Sheth, R. K., \& Tormen, G., 2002, MNRAS, 329, 51

Silk, J., \& Rees, M. J., 1998, A\&A, 331L, 1

Springel, V., Di Matteo, T., \& Hernquist, L., 2005a, MNRAS, in press, astro-ph/0411108

Springel, V., Di Matteo, T., \& Hernquist, L., 2005b, ApJ, in press, astro-ph/0409436

Springel, V. et al., 2005c, astro-ph/0504097

Tremaine, S. et al., 2002, ApJ, 574, 740

Wyithe, J. S. B., \& Loeb, A., 2002, ApJ, 581, 886

Wyithe, J. S. B., \& Loeb, A., 2003, ApJ, 595, 614

Wyithe, J. S. B., \& Loeb, A., 2005, ApJ, 621, 95

Yang, X., Mo, H. J., \& van den Bosch, F. C., 2003, MNRAS, 339, 1057 